\title{
Declaration of Hawaii
}

\author{
World Psychiatric Association
}

\section{Editor's introduction}

We are printing in full the text of the Declaration of Hawaii, a Code of Ethics adapted by the World Psychiatric Association at its meeting in Honolulu in August 1977. The text is followed by a brief commentary from Dr Clarence Blomquist, the person responsible for drawing up the draft code. Dr Blomquist argues that the main function of a code of this kind is to supply general guidelines which will alert psychiatrists to the ethical problems and moral traps of their profession.

\section{DECLARATION OF HAWAII}

Ever since the dawn of culture ethics has been an essential part of the healing art. Conflicting loyalties for physicians in contemporary society, the delicate nature of the therapist-patient relationship, and the possibility of abuses of psychiatric concepts, knowledge and technology in actions contrary to the laws of humanity, all make high ethical standards more necessary than ever for those practising the art and science of psychiatry.

As a practitioner of medicine and a member of society, the psychiatrist has to consider the ethical implications specific to psychiatry as well as the ethical demands on all physicians and the societal duties of every man and woman.

A keen conscience and personal judgement is essential for ethical behaviour. Nevertheless, to clarify the profession's ethical implications and to guide individual psychiatrists and help form their consciences, written rules are needed.

Therefore, the General Assembly of the World Psychiatric Association has laid down the following ethical guidelines for psychiatrists all over the world.

I The aim of psychiatry is to promote health and personal autonomy and growth. To the best of his or her ability, consistent with accepted scientific and ethical principles, the psychiatrist shall serve the best interests of the patient and be also concerned for the common good and a just allocation of health resources.

To fulfil these aims requires continuous research and continual education of health care personnel, patients and the public.
2 Every patient must be offered the best therapy available and be treated with the solicitude and respect due to the dignity of all human beings and to their autonomy over their own lives and health.

The psychiatrist is responsible for treatment given by the staff members and owes them qualified supervision and education. Whenever there is a need, or whenever a reasonable request is forthcoming from the patient, the psychiatrist should seek the help or the opinion of a more experienced colleague.

3 A therapeutic relationship between patient and psychiatrist is founded on mutual agreement. It requires trust, confidentiality, openness, co-operation and mutual responsibility. Such a relationship may not be possible to establish with some severely ill patients. In that case, as in the treatment of children, contact should be established with a person close to the patient and acceptable for him or her.

If and when a relationship is established for purposes other than therapeutic, such as in forensic psychiatry, its nature must be thoroughly explained to the person concerned.

4 The psychiatrist should inform the patient of the nature of the condition, of the proposed diagnostic and therapeutic procedures, including possible alternatives, and of the prognosis. This information must be offered in a considerate way and the patient be given the opportunity to choose between appropriate and available methods.

5 No procedure must be performed or treatment given against or independent of a patient's own will, unless the patient lacks capacity to express his or her own wishes or, owing to psychiatric illness, cannot see what is in his or her best interest or, for the same reason, is a severe threat to others.

In these cases compulsory treatment may or should be given, provided that it is done in the patient's best interests and over a reasonable period of time, a retroactive informed consent can be presumed and, whenever possible, consent has been obtained from someone close to the patient.

6 As soon as the above conditions for compulsory treatment no longer apply the patient must be released, unless he or she voluntarily consents to further treatment. 
Whenever there is compulsory treatment or detention there must be an independent and neutral body of appeal for regular inquiry into these cases. Every patient must be informed of its existence and be permitted to appeal to it, personally or through a representative, without interference by the hospital staff or by anyone else.

7 The psychiatrist must never use the possibilities of the profession for maltreatment of individuals or groups, and should be concerned never to let inappropriate personal desires, feelings or prejudices interfere with the treatment.

The psychiatrist must not participate in compulsory psychiatric treatment in the absence of psychiatric illness. If the patient or some third party demands actions contrary to scientific or ethical principles the psychiatrist must refuse to co-operate. When, for any reason, either the wishes or the best interests of the patient cannot be promoted, he or she must be so informed.

8 Whatever the psychiatrist has been told by the patient, or has noted during examination or treatment, must be kept confidential unless the patient releases the psychiatrist from professional secrecy, or else vital common values or the patient's best interest makes disclosure imperative, In these cases, however, the patient must be immediately informed of the breach of secrecy.

9 To increase and propagate psychiatric knowledge and skill requires participation of the patients. Informed consent must, however, be obtained before presenting a patient to a class and, if possible, also when a case history is published, and all reasonable measures be taken to preserve the anonymity and to safeguard the personal reputation of the subject.

In clinical research, as in therapy, every subject must be offered the best available treatment. His or her participation must be voluntary, after full information has been given of the aims, procedures, risks and inconveniences of the project, and there must always be a reasonable relationship between calculated risks or inconveniences and the benefit of the study.

For children and other patients who cannot themselves give informed consent this should be obtained from someone close to them.

IO Every patient or research subject is free to withdraw for any reason at any time from any voluntary treatment and from any teaching or research programme in which he or she participates. This withdrawal, as well as any refusal to enter a programme, must never influence the psychiatrist's efforts to help the patient or subject.

The psychiatrist should stop all therapeutic, teaching or research programmes that may evolve contrary to the principles of this Declaration.

\section{Commentary}

\section{Clarence D D Blomquist \\ Karolinska Institute, Stockholm, Sweden}

When the World Psychiatric Association asked me, early in 1976, to draft an international code of ethics for psychiatry, the Declaration of Hawaii, I was a visiting scholar at the Institute of Society, Ethics and the Life Sciences in Hastings-onHudson, New York. In this stimulating and really scholar.y atmosphere I had become aware of the sometimes diverse approaches to medical ethics and the physician/patient relationship in Europe and the US ${ }^{1}$. In Europe we stick to the Oath of Hippocrates, and our ethic rests on a benevolent paternalism. We really care for our patients, but nothing of the decision is left to them. The doctor takes over all responsibility. In the US medical ethics is more connected to the Constitution than to Hippocratic ethic and one speaks in terms of constitutional or human rights. Medical decisions rest more with the patient or his/her legal proxy. Ethics becomes a branch of law and this often leads to bureaucracy and a frequent suing of the doctor for malpractice.

My endeavour when drafting the Declaration was to avoid these extremes and find some balance in between. I tried to gain more concern for the patients' autonomy and right to participate in decisions about their own lives and health but to avoid a rigid legalistic system and to give place for man's legitimate need for trust, confidence and care.

The relationship between the patients and their psychiatrists then becomes an agreement between two autonomous parties, requiring 'trust, confidentiality, openness, co-operation and mutual responsibility'. So the aim of psychiatry could be said to be to promote not only health but also 'personal autonomy and growth'.

But is there really need for a code of ethics for psychiatry ? Yes, I think so. Psychiatry has many specific features and problems unknown or at least less evident in other branches of medicine, and I hope most of them are covered by the Declaration. Our Russian colleagues are said to misuse psychiatry for political reasons. This type of misuse is well covered by the paragraphs 5 to 7 . But there are other kinds of misuse and other ethical problems in psychiatry today.

We doctors, psychiatrists or not, no longer go on declaring our sole duty being towards the present patient. We have, and we are getting more and more, conflicting loyalties; towards the patient, presumptive patients, society and the common good.

The great problems I find, however, not in involuntary treatment and incarceration, but in the inordinate spreading of different psychotherapies, some reasonable and good, some less so, and the 